\title{
ESTUDOS SOBRE A EPIDEMIOLOGIA DA DOENÇA DE CHAGAS NO CEARÁ XX - ECOLOGIA DE TRIATOMINEOS EM RUSSAS *
}

\author{
Joaquim Eduardo de Alencar, Otávio Ferreira Bezerra e \\ Oswaldo Freire de Faria Filho.
}

\begin{abstract}
Foi escolhida para demolição, no Municipio de Russas, território do Triatoma braziliensis, uma casa borrifada 104 dias antes com BHC. Na área - a Fazenda Boa Vista - resisdem 250 habitan. tes; a taxa de infeccão humana é 7,7\% (imunofluorescência indireta) e a de cães é $9,1 \%$ (xeno).

A captura prévia na área revelou $171 \mathrm{~T}$. braziliensis $(46,8 \%)$ infectados e raros Triatoma pseudo maculata (5). Na casa havia um habitante com teste de imunofluorescência positivo e de 26 T. braziliensis capturados nenhum revelou infeccão.

Durante a demolição foram capturados 1 rato infectado pelo Trypanosoma cruzi e 104 triatomineos, $189,4 \% \mathrm{~T}$. pseudomaculata e $10,6 \% \mathrm{~T}$. brazieliensis); havia R. nasutus infectado no galinheiro.

Exemplares de T. pseudomaculata infectados foram encontrados no teto $\left(0,6\right.$ por $\left.\mathrm{m}^{2}\right)$ e nas paredes externas e internas da sala $\left(0,8\right.$ por $\left.\mathrm{m}^{2}\right)$; ao teste de precipitina revelaram acentuada antropo filia.

Concluiu-se que numa casa normalmente habitada por T. braziliensis, após a borrifação, houve substituição dessa espécie por uma população de T. pseudomaculata, numa área com razoável antropofilia e taxa de infecção suficiente para a transmissão.
\end{abstract}

\section{INTRODUÇÃO}

Desde 1959 vem sendo visitada uma área rural do município de Russas, em torno da cidade sede do município, verificando-se altas taxas de infecção humana $(11,6 \%)^{1}$ de cães $(14,7 \%)$ e gatos $(24,5 \%)^{2}$ de triatomíneos $(19,9 \%)^{3}$ e de outros animais $(22,1)^{1}$.

A fim de proceder a um estudo longitudinal, selecionamos a micro-área da Fazenda Boa Vista, na qual foi constatada $7,7 \%$ de infecção de triatomineos. Foi observada também a infecção de outros animais: Rattus rattus frugivorus, Rattus rattus alexandrinus e Didelphis azarae ${ }^{1}$.

No município de Russas o triatomíneo dominante é o Triatoma braziliensis; o T. pseudo maculata é secundário; são encontrados também raros exemplares de $P$. megistus e $R$. nasustus ${ }^{3}$.

Constituindo-se o município - a área rural próximo à sede e a Fazenda Boa Vista - áreas de maior prevalência do principal transmissor do T. cruzi no Ceará - o T. braziliensis ${ }^{4}$, é a área que vém sendo periodicamente expurgada e foi por este motivo a área selecionada para estudos de prevalência.

Em vista de que foi recentemente expurgado a área com BHC, conviria selecionar uma casa para demolição a fim de testar a validade do expurgo com medida profilática real.

\section{MATERIAL E MÉTODO}

Em uma área selecionada por suas caracteristicas (Tabela I) foi estudada a Fazenda Boa Vista, localizada no município de Russas. O clima é quente e úmido. As casas estudadas (40) abrigam 250 habitantes em 270 aposentos. Predorninam as casas de barro (taipa ou sopapo) sem reboco, teto de telha e piso de barro batido. Existem 132 mamíferos dentro das casas (cães, gatos, coelhos e saguis) e 752 no peridomicilio (ovelhas, cabras, porcos e coelhos).

Na captura prévia foram coletados 176 triatomíneos em 1977 (Tabela II), sendo mais prevalente o Triatoma braziliensis (97,3\%).

* Trabalho do Centro de Ciências da Saúde da Universidade Federal do Ceará e da SUCAM, realizado com auxilio do CNPq. 
Tabela N? 1

Boa Vista - Município de Russas - Estado do Ceará - Brasil

Dados da localidade: Antes da borrifação - Julho - 1977

Altitude média . . . . . . . . . . . . . . . . . . . . . 101,00 m

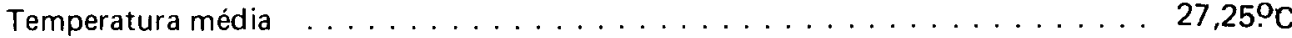

Unidade média . . . . . . . . . . . . . . . . . . . . . . . 70,00\%

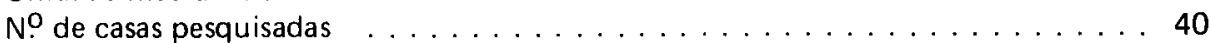

№ de habitantes . . . . . . . . . . . . . . . . . . . . . . . . 250

Habitantes / casa . . . . . . . . . . . . . . . . . . . 6.25

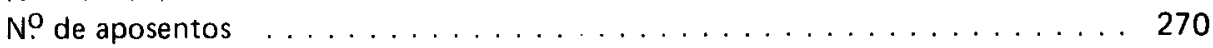

Habitante / aposento . . . . . . . . . . . . . . . . . . . 0,93

Casas com paredes de barro com reboco $\ldots \ldots \ldots \ldots \ldots \ldots \ldots \ldots \ldots \ldots$

Casas com paredes de barro sem reboco . . . . . . . . . . . . . 20

Casas com paredes de tijolo com reboco $\ldots \ldots \ldots \ldots \ldots \ldots \ldots$

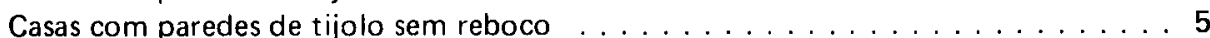

Casas com tetos de telha . . . . . . . . . . . . . . . . . . 39

Casas com tetos de palha $\ldots \ldots \ldots \ldots \ldots \ldots \ldots \ldots \ldots$

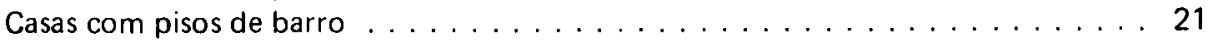

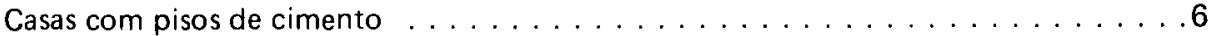

Casas com pisos de tijolo . . . . . . . . . . . . . . . . 13

№ de mamíferos no interior do domicílio (132):
a) cães

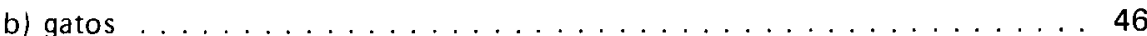

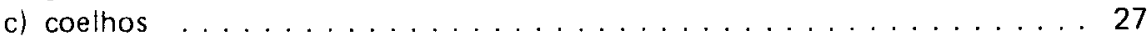

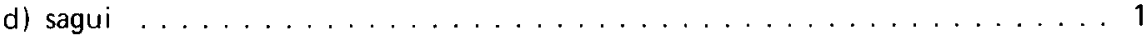

de mamíferos no peridomicílio (752)
a) cabras
b) coelhos
c) ovelhas
d) porcos

№ de aves no interior do domicílio (15)
a) galinhas
b) papagaios

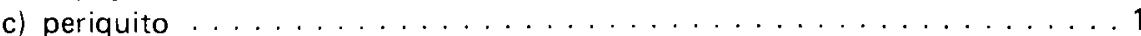

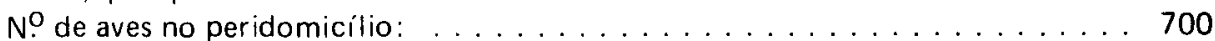

№ de triatomineos capturados (176):

a) no interior do domicílio:

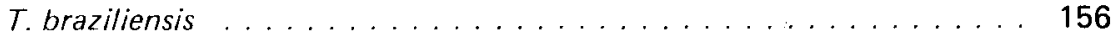

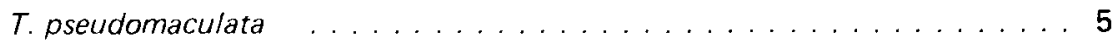

b) no peridomicílio:

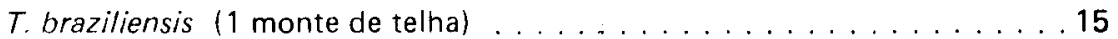

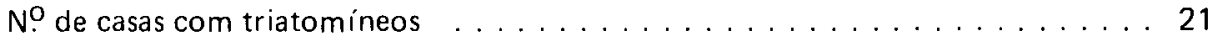

NO de casas com triatomíneos infectados pelo $T$. cruzi . . . . . . . . . . . . 19

$\mathrm{N}^{\mathrm{O}}$ de ecótopos pesquisados no interior do domicílio . . . . . . . . . . . 270

$\mathrm{N}^{\mathrm{O}}$ de ecótpos no interior do domicílio $\mathrm{c} /$ triatomíneos $\ldots \ldots \ldots \ldots$

No de ecótopos no interior do domicílio com triatomíneos

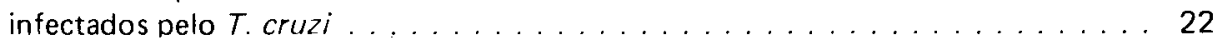

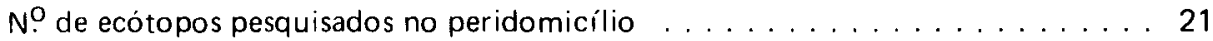

No de ecótopos no peridomicílio com triatomíneos infectados pelo $T$. cruzi . . . . 1

Espécies de triatomíneos examinados:

a) T. braziliensis examinados (capturados no intradomicilio) . . . . . . 156

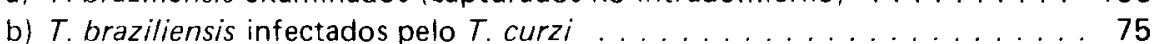

c) T. pseudomaculata examinados (capturados no intradomicílio 5

d) $T$. pseudomaculata infectado pelo $T$. cruzi . . . . . . . . . . . . . 0

e) T. braziliensis examinados (capturados no peridomicílio . . . . . . . 15

f) $T$. braziliensis infectados pelo $T$. cruzi ................ 5 
Tabela II

Estudos sobre a epidemiologia da doença de Chagas no Ceará Município de Russas - Fazenda Boa Vista - Triatomíneos capturados

\begin{tabular}{|c|c|c|c|c|c|c|c|c|}
\hline \multirow{3}{*}{ E SPÉ C I E } & \multicolumn{2}{|c|}{$1973 / 1974$} & \multicolumn{2}{|c|}{1977} & \multicolumn{4}{|c|}{1978 - Casa demolida } \\
\hline & \multirow[b]{2}{*}{ Capturados } & \multirow[b]{2}{*}{$\%$} & \multirow[b]{2}{*}{ Capturados } & \multirow[b]{2}{*}{$\%$} & \multicolumn{2}{|c|}{ Antes } & \multicolumn{2}{|c|}{ Durante } \\
\hline & & & & & $\begin{array}{l}\text { Captu- } \\
\text { rados }\end{array}$ & $\%$ & $\begin{array}{c}\text { Captu- } \\
\text { rados }\end{array}$ & $\%$ \\
\hline Triatoma braziliensis & 379 & 93,5 & 171 & 97,3 & 06 & 100,0 & 11 & 10,6 \\
\hline Triatoma pseudomaculata & 22 & 5,4 & 05 & 2.7 & - & - & 93 & 89,4 \\
\hline Panstrongy/us megistus & 04 & 1,1 & - & - & - & - & - & - \\
\hline Rhodnius nasutus & - & - & - & - & - & - & $21^{*}$ & - \\
\hline T. braziliensis & & & & & & & $4 \%$ & \\
\hline TOTAL & 405 & 100,0 & $176 \quad 10$ & 0,00 & 06 & 100,0 & 129 & 100,0 \\
\hline
\end{tabular}

* No peridomicílio

Em Boa Vista a infecção Humana é de $7,7 \%$ (Tabela III), verificada pelo teste de imunofluorescência indireta. Essa taxa não difere muito das observadas nos sítios vizinhos (Tabela IV). A distribuição etária mostra que de 10 a 34 anos não se observa resultado positivo (Tabela $V$ ).

A infecção de animais domésticos é elevada (cães e gatos), verificando-se no entanto IIma diminuição de prevalência de 1973 para 1977 (Tabela VI).

Trata-se assim de uma área endêmica de doença de Chagas, com prevalência do $T$. braziliensis.
A casa 130 selecionada para demolição, com aquiescência de seu proprietário, é indicada na Tabela VII. Borrifada com BHC a 17.11.1977, nela residem 2 pessoas em 3 aposentos, sendo uma das pessoas TIF positiva. Foram capturados $6 T$. braziliensis dos quais 3 estavam infectados com $T$. cruzi. Foram realizados 2 xenos em cão e gato, com resultado negativo.

A área do piso é $44 \mathrm{~m}^{2}$, do te to é $56 \mathrm{~m}^{2}$ e das paredes internas $82 \mathrm{~m}^{2}$. A temperatura média 31,4 ? $\mathrm{C}$, e a umidade média $61,8 \%$.

A casa foi fotografada e medidas as paredes, o piso e o teto de cada um dos 3 comparti-

Tabela III

Estudos sobre a epidemiologia da doença de Chagas

Estado do Ceará - Município de Russas Infecção Humana - 1959/1978

\begin{tabular}{|c|c|c|c|c|}
\hline Área Estudada & Ano & $\begin{array}{l}\text { Teste } \\
\text { Diagnóstico }\end{array}$ & $\begin{array}{l}\text { No de } \\
\text { Provas }\end{array}$ & $\begin{array}{l}\% \text { de } \\
\text { Positivas }\end{array}$ \\
\hline \multirow[t]{4}{*}{ Cidade e Sítios vizinhos } & 1959 & $\mathrm{RFC}^{* *}$ & 146 & 11,6 \\
\hline & 1970 & & & \\
\hline & 1974 & $\mathrm{RFC}^{* *}$ & 194 & 9,8 \\
\hline & 1975 & $\operatorname{RFC}^{* *}$ & 340 & 10,5 \\
\hline Alguns Sítios * & 1978 & TIF *** & 409 & 7,6 \\
\hline Fazenda Boa Vista & 1978 & TIF *** & 169 & 7,7 \\
\hline
\end{tabular}

* Boa Vista, Várzea Alegre, Melancia, Ingá, Malhadinha

* Reação de Guerreiro e Machado

*** Teste de Imunofluorescência indireta 
Tabela IV

Estudos sobre a epidemiologia da doença de Chagas no Ceará TIF * Realizados no município de Russas

1978

\begin{tabular}{lccr}
\hline Sítios Examinados & No de Provas & Positivas & $\%$ \\
\hline Boa Vista & 169 & 13 & 7,7 \\
Várzea Alegre & 31 & 2 & 6,5 \\
Melancia & 89 & 5 & 10,1 \\
Ingá & 98 & 2 & 5,1 \\
Malhadinha & 22 & 31 & 9,1 \\
\hline T O T A L & 409 & 3,6 \\
\hline
\end{tabular}

* Testes de imunofluorescência

Tabela V

Estudos sobre a epidemiologia da doença de Chagas no Ceará Fazenda Boa Vista - Município de Russas Testes de Imunofluorescência Indireta - 1978

\begin{tabular}{|c|c|c|c|c|}
\hline \multirow{2}{*}{\multicolumn{2}{|c|}{$\begin{array}{c}\text { Grupos } \\
\text { de } \\
\text { Idade }\end{array}$}} & \multicolumn{2}{|c|}{$T$} & $F$ \\
\hline & & \multirow{2}{*}{$\begin{array}{c}\begin{array}{c}\text { Total } \\
\text { Examinado }\end{array} \\
16\end{array}$} & \multirow{2}{*}{ Positivo } & \multirow{2}{*}{ Negativo } \\
\hline 0 & -4 & & & \\
\hline 5 & -9 & 23 & 1 & 22 \\
\hline 10 & -14 & 23 & - & 23 \\
\hline 15 & $-\quad 19$ & 22 & - & 22 \\
\hline 20 & -24 & 11 & - & 11 \\
\hline 25 & -29 & 6 & - & 6 \\
\hline 30 & -34 & 10 & - & 10 \\
\hline 35 & -39 & 4 & 1 & 3 \\
\hline 40 & -44 & 6 & 2 & 4 \\
\hline 45 & -49 & 13 & 2 & 11 \\
\hline 50 & -54 & 9 & 1 & 8 \\
\hline 55 & -59 & 4 & 1 & 3 \\
\hline 60 & -64 & 5 & 1 & 4 \\
\hline 65 & -69 & 5 & - & 5 \\
\hline 70 & -74 & 5 & 1 & 4 \\
\hline & -79 & 3 & - & 3 \\
\hline \multirow[t]{3}{*}{80} & $a^{+}$ & 4 & 1 & 3 \\
\hline & TOTAL & 169 & 13 & 156 \\
\hline & $\%$ & 100 & 7,7 & 92,3 \\
\hline
\end{tabular}


mentos. A demolição foi realizada metro por metro, a começar pelas paredes da sala e terminando pelo teto. Os torrões de barro eram desfeitos $e$ as telhas revistadas uma a uma; os animais encontrados era capturados para identificação - anotando-se a sua exata localização.
Foram medidas a temperatura e a umidade relativa em cada etapa. Os triatomíneos eram capturados e postos em recipientes de vidro, separadamente por localização. Eram posteriormente classificados, examinados quanto à infecção pelo $T$. cruzi e os hábitos alimentares.

Tabela VI

Estudos sobre a epidemiologia da doença de Chagas no Ceará Fazenda Boa Vista - Município de Russas

Xeno de Cães e Gatos

\begin{tabular}{lccc}
\hline $\begin{array}{c}\text { Animais } \\
\text { Domésticos } \\
\text { Examinados }\end{array}$ & \multicolumn{3}{c}{ D A T A } \\
\cline { 2 - 4 } CÃES & & & 1977 \\
Examinados & 71 & 44 \\
Positivos & 11 & 4 \\
$\%$ & 15,5 & 9,1 \\
\hline GATOS & & \\
Examinados & 32 & 21 \\
Positivos & 17 & 1 \\
$\%$ & 53,1 & 4,8 \\
\hline TOTAL & & 65 \\
Examinados & 103 & 5 \\
Positivos & 28 & 7,7 \\
\hline
\end{tabular}


Tabela VII

Boa Vista - Município de Russas - Estado do Ceará - Brasil

Dados da casa n: 130 depois da borrifação - 17/11/1977

Casa demolida de primeiro (19) de março de 1978

Habitantes . . . . . . . . . . . . . . . .

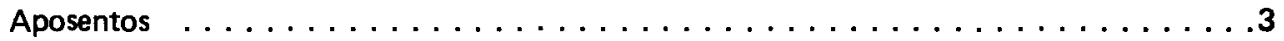

Teperatura média durante a demolição $\ldots \ldots \ldots \ldots \ldots \ldots \ldots \ldots \ldots \ldots \ldots \ldots$ 31,409 $\mathrm{C}$

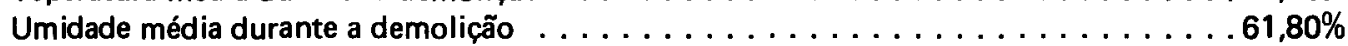

№ de triatomíneos capturados no domicílio . . . . . . . . . . . . . . . . . 104

№ de triatomíneos examinados . . . . . . . . . . . . . . . . . . . . . 101

№ de triatomíneos infectados pelo $T$. cruzi $\ldots \ldots \ldots \ldots \ldots \ldots \ldots \ldots \ldots \ldots \ldots$ (81,2\%)

Espécies de Triatomineos capturados:

T. pseudomaculata (90): adultos examinados .........8 8

adultos infectados pelo $T$. cruzi . . . . 4

ninfas examinadas .......... 82

ninfas infectadas pelo $T$. cruzi ..... 69

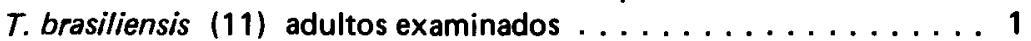

adultos infectados pelo $T$. cruzi. . . . . . . 1

ninfas examinadas ........... 10

ninfas infectadas pelo $T$. cruzi .............8

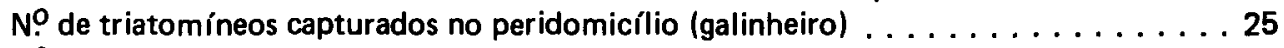

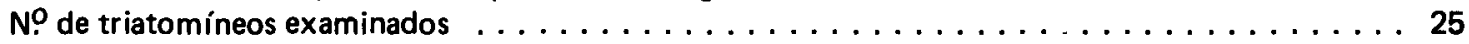

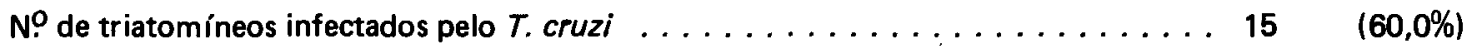

Espécies de triatomíneos capturados:

R. nasutus 21:

adultos examinados . . . . . . . 7

adultos infectados pelo $T$. cruzi ..... 6

ninfas examinadas ........... 14

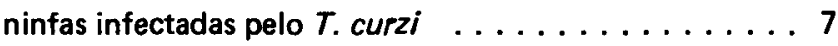

T. braziliensis (4): $\quad$ ninfas examinadas . . . . . . . 4

ninfas infectadas pelo $T$. cruzi ........... 3

\section{RESULTADOS}

Animais encontrados - A Tabela VIII mostranos que existiam dentro da casa 24 vertebrados dos quais 20 são habitualmente sugados por triatomíneos. Foram observados, mas não contados, aracnídeos e insetos dentro da casa: quarto, sala, cozinha e teto (baratas, besouros, cupins e formiga). Foram capturados 129 exemplares de triatomíneos, sendo 25 capturados no peridomicílio e 104 dentro da casa (Tabela II, dos quais 101 foram examinados (Tabela IX).

Infecção pelo $T$. cruzi - Um dos ratos capturados (Rattus rattus frugivorus) estava infectado, bem como uma das pessoas residentes. Dos triatomíneos examinados $77 \%$ estavam infectados, sendo maior a taxa observada nas paredes da sala (Tabela 8). Também os triatomíneos capturados no galinheiro apresentaram elevada taxa de infecção.

Estádios evolutivos - Dada a abundância das fontes de alimentação observadas, a colônia de triatomíneos mostrava grande número de estádios ninfais: de 126 examinados somente 16 eram adultos.

Distribuição por ecótopos - Os triatomíneos capturados, excetuando-se os de peridomicílio (galinheiro), foram nas paredes da sala $(56 \%)$ e no teto $(44 \%)$. Levando em conta o total geral, teremos as seguintes porcentagens:

a) no galinheiro . . . . . . 19,3\%

b) nas paredes da sala $\ldots \ldots \ldots 45,0 \%$

c) no teto $\ldots \ldots \ldots \ldots \ldots \ldots, 35,7 \%$ 
Tabela VIII

Estudos sobre a epidemiologia da doença de Chagas no Ceará.

Animais existentes na casa 130 - Fazenda Boa Vista - Município de Russas - 1978

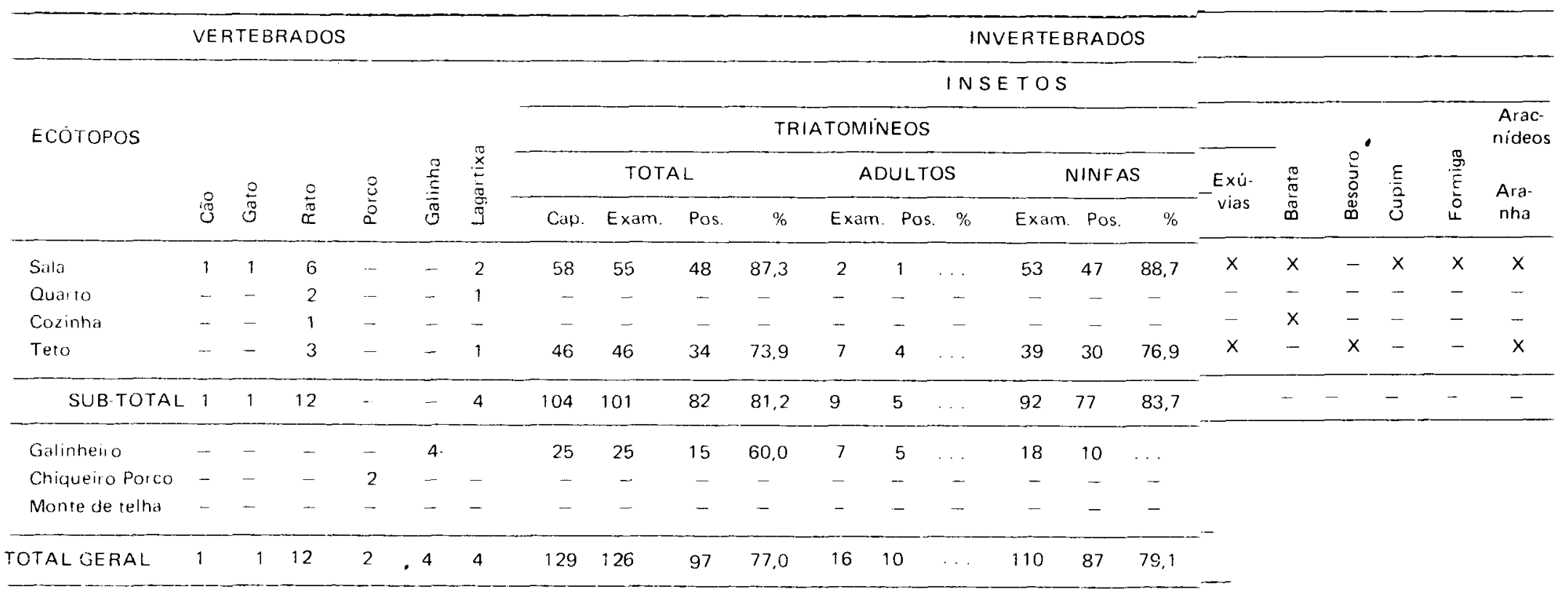


Tabela IX

Estudos sobre a epidemiologia da doença de Chagas no Ceará - Fazenda Boa Vista Triatomíneos capturados em casa demolida (03 a 05/03/1978) e borrifação com RFC 104 dias antes (17/11/1977)

\begin{tabular}{|c|c|c|c|c|c|c|c|c|c|c|c|}
\hline \multirow{2}{*}{ ECÓTOPOS } & & \multirow{2}{*}{ ESPÉCIES } & \multicolumn{4}{|c|}{ ADULTOS } & \multicolumn{2}{|c|}{ NINFAS } & \multicolumn{3}{|c|}{ TOTAL } \\
\hline & & & Exam. & Pos. & $\%$ & Exam. & Pos. & $\%$ & Exam. & Pos. & $\%$ \\
\hline SALA-Parede externa & 43 & T.pseudo maculata & 1 & 1 & . & 40 & 36 & 90,0 & 41 & 37 & 90,2 \\
\hline SALA-Parede interna & 11 & T.pseudo maculata & 1 & - & - & 9 & 8 & . . & 10 & 8 & $\cdots$ \\
\hline TETO-da sala & 37 & T.pseudo maculata & 5 & 3 & $\cdots$ & 32 & 25 & 78,1 & 37 & 28 & 75,7 \\
\hline TETO-do quarto & 1 & T.pseudo maculata & - & - & - & 1 & - & - & 1 & - & - \\
\hline TETO-da cozinha & 1 & T.pseudo maculata & 1 & - & - & - & - & - & 1 & - & - \\
\hline SUB-TOTAL & 93 & - & 8 & 4 & - & 82 & 69 & 84,1 & 90 & 73 & 81,1 \\
\hline SALA-parede externa & 1 & T. braziliensis & - & - & - & 1 & - & - & 1 & - & - \\
\hline SALA-Parede interna & 3 & T. braziliensis & - & - & - & 3 & 3 & $\ldots$ & 3 & 3 & . \\
\hline TETO-da sala & 5 & T. braziliensis & 1 & 1 & $\ldots$ & 4 & 3 & $\ldots$ & 5 & 4 & $\because$ \\
\hline TETO-do quarto & 2 & T. braziliensis & - & - & - & 2 & 2 & $\cdots$ & 2 & 2 & $\cdots$ \\
\hline SUB-TOTAL & 11 & - & 1 & 1 & & 10 & 8 & $\cdots$ & 11 & 9 & $\cdots$ \\
\hline TOTAL GERAL & 104 & - & 9 & 5 & . & 92 & 77 & 83,7 & 101 & 82 & 81,2 \\
\hline
\end{tabular}


Espécies capturadas - As tabelas 11 e IX mostram a distribuição por espécie na área e na casa demolida. Verificamos que a maior prevalência na área é do $T$. braziliensis e na casa demolida era a única espécie encontrada antes da demolição, dentre as quatro espécies de triatomíneos encontrados na Fazenda Boa Vista (Gráfico la).

Durante a demolição foram econtrados na casa $93 \mathrm{~T}$. pseudomaculata e $11 \mathrm{~T}$. braziliensis, e que representa a inversão da prevalência (Gráfico (b); a primeira é a espécie de menor prevalência na região, tendo em conta as duas espécies focalizadas, e passou a ser na casa demolida a de maior prevalência. Não foram encontrados
$P$. megistus e o $R$. nasatus foi encontrado somente no galinheiro da casa demolida.

Por espécie e ecótopo, verificamos que o T. pseudomaculata foi mais abundante nas paredes externas da sala e no teto da sala, enquanto o $T$. braziliensis foi mais abundante nas paredes internas e no teto da sala.

A taxa de infecção de ambas as espécies é praticamente idêntica (Gráfico 2). Os ecótopos mais infectados foram as paredes e o teto da sala, como também mais elevadas as taxas de infecção. As ninfas apresentaram-se mais infectadas que os adultos (Gráfico 3 ): $76,2 \%$ contra $5,0 \%$, em 101 triatomíneos examinados.

\section{Gráfico 1}

Estudo sobre a epidemiologia da doença de Chagas No estado do Ceará - Ecologia de triatomineos em Russas

\section{Triatomíneos capturados na casa 130 do Sítio Boa Vista $1977-1978$ \\ Infectados por espécie e fases evolutivas}
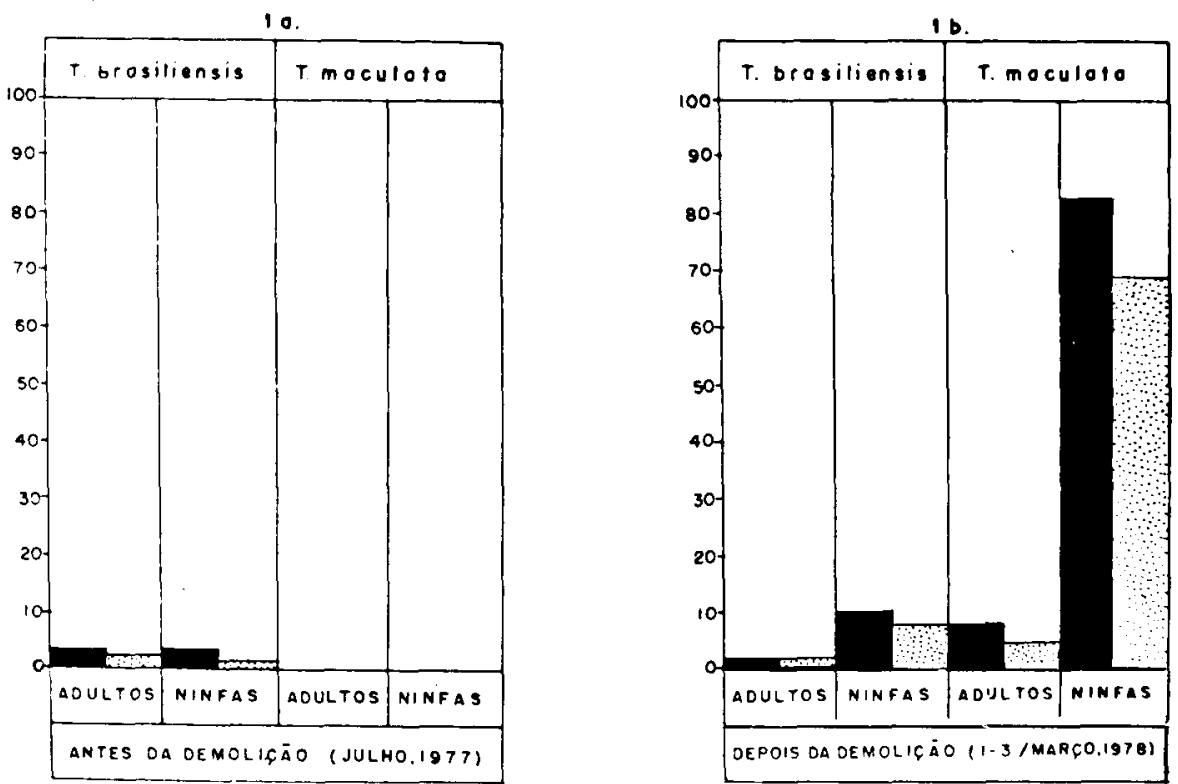

LE GENOA: EXAMINAOOS positivos 
Gráfico 2

Estudos sobre a epidemiologa da doença de Chagas no estado do Ceará - Ecologia de triatomíneos em Russas

Triatomíneos capturados: Examinados e positivos Sítio Boa Vista, Março - 1978

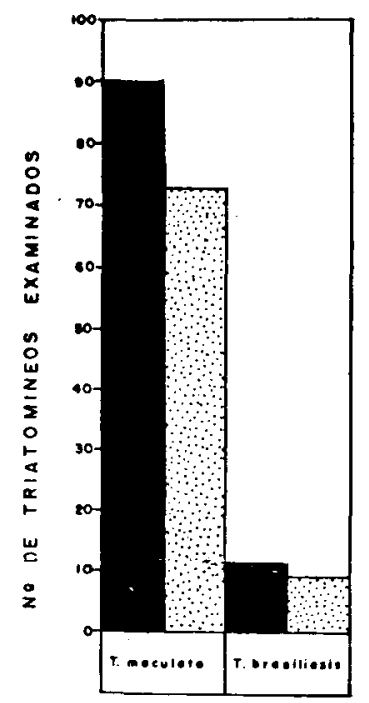

LEGENDA:

Examinados Positivos

Gráfico 3

Estudos sobre a epidemiologia da doença de Chagas no estado do Ceará - Ecologia de triatomíneos em Russas

Triatomíneos capturados (total: adultos e ninfas) Sítio Boa Vista, Março - 1978

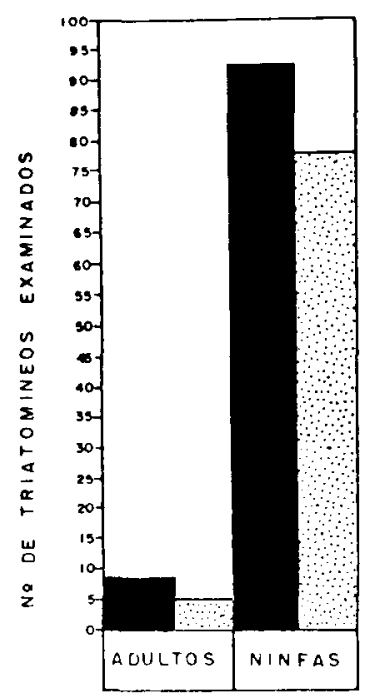

LEGENDA:

Examinados Positivos 


\section{DISCUSSÃO}

Circulação do T. cruzi dentro da habitação Dentro da habitação havia 26 vertebrados, incluindo os dois habitantes do domicílio e 129. triatomíneos disputavam a opção, o que significa 5 triatomíneos para cada um. Como a taxa de infecção desses triatomíneos era de $77,0 \%$ significava que 1 em 5 estava infectado, ou seja, um infectado para cada vertebrado. Desses, 2 estavam infectados, o que signifca que a oportunidade de infecção dos triatomíneos era de $40 \%$ em cada repasto e de $20 \%$ das vezes cada vertebrado se infectaria, caso um deles chegasse ao climax e dejetasse. Vamos então considerar como máxima a probabilidade de transmissão do $T$. cruzi entre hospedeiros vertebrados.

Essa probabilidade existiu pelo menos em relação ao rato infectado, pois nas provas de precipitina para verificar os hábitos alimentares, $43 \%$ foram positivas para antígeno de rato; as demais referem-se a cão, gato e galinha. Constatamos que os triatomíneos capturados na casa demolida não apresentaram positividade para antígeno do homem (Tabela 10).

Inversão da prevalência das duas espécies Antes da borrifação, a acaptura revelava na casa apenas o $T$. braziliensis, espécie mais prevalente no município e na área; após a borrifação a situação modificou-se e passou o $T$. pseudomaculata a ser $89,4 \%$ dos exemplares capturados, contra $10,6 \%$ do $T$. braziliensis. Isso leva-nos a pensar em uma maior capacidade de resistência da espécie, abrigando-se nas paredes externas e no teto ou maior resistência diante do inseticida (Gráfico 1).

Ecótopos de resistência - Os ecótopos onde foram encontrados os triatomíneos são o interior das paredes, pelos lados externo e interno. principalmente na sala, e no teto. Não foi difícil encontrar um abrigo livre de inseticida e também foi fácil encontrar a oportunidade de sugar o roedor, o cão, o gato sem necessitar entrar em contato com a superfície borrifada da parede.

\section{CONCLUSÃO}

A casa demolida constituia um nicho onde o T. cruzi circulava bem, infectando o homem, roedor e triatomíneos em taxas elevadas;

Os triatomíneos observados numa casa por meio dos métodos habituais da captura é apenas uma pequena fração da colônia completa $(5,8 \%)$;

Os triatomíneos em casa borrifada com BHC pelos métodos tradicionais, resistem em ecótopos vários, sendo a parede externa e o teto da sala para $T$. pseudomaculata e a parede interna e o teto da sala para o $T$. braziliensis,

O T. pseudomaculata apresenta-se mais resistente que o $T$. braziliensis, substituindo-o na ordem de prevalência na casa observada.

Tabela X

Estudos sobre a epidemiologia da doença de Chagas no Ceará Provas de precipitina (112) em fezes de triatomineos - 1978 Boa Vista ( 9 casas)

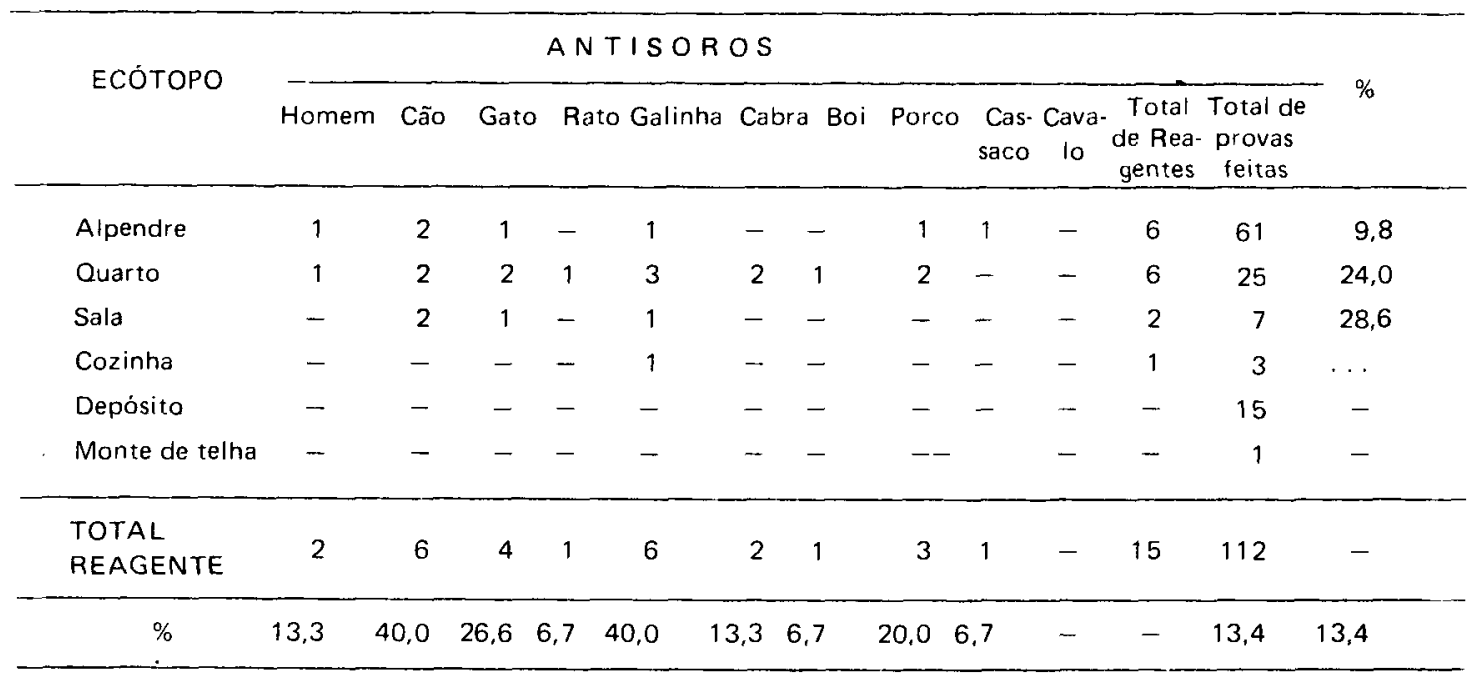


Tabela XI

Estudos sobre a epidemiologia da doença de Chagas no Ceará

Município de Russas - Fazenda Boa Vista - Casa No 130

Provas de precipitina com triatomíneos - 1977

\begin{tabular}{|c|c|c|c|c|c|c|c|c|c|c|c|}
\hline EСÓTOPO & Homen & & Cão Gato & Ratc & ○ Gali- & Cabra & Boi P.orco & $\begin{array}{l}\text { Marsu- } \\
\text { pião }\end{array}$ & 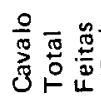 & $\begin{array}{l}\text { Total } \\
\text { Reagentes }\end{array}$ & es $\%$ \\
\hline Parede interna $(3 \mathrm{~T} . \mathrm{b} .+10 \mathrm{~T} . \mathrm{m})$. & $1-$ & 1 & 7 & 1 & 2 & - & - & - & -13 & 7 & 53,8 \\
\hline Teto (5 T.b. +39 T.m.) & - & 2 & 23 & 102 & 22 & - & - & - & -46 & 33 & 71,7 \\
\hline Parede externa (42 T.m.) & - & 11 & 34 & 302 & 26 & - & - & - & -42 & 38 & 90,5 \\
\hline Galinheiro (5 T.b. + R.n.) & - & 8 & 14 & 21 & 16 & - & - & - & -25 & 22 & 88,0 \\
\hline TOTAL & - & 22 & 78 & 436 & 66 & - & - & - & -126 & 100 & - \\
\hline$\%$ & - & 22 & 78 & 43 & 66 & - & - & - & - & 79,4 & 79,4 \\
\hline
\end{tabular}

T.B. - T. braziliensis

T.m. - T. pseudomaculata

R.n. - R. nasutus

\section{SUMMARY}

A house sprayed 104 days before with BHC was selected for demolition. It was sited in the municipality of Russas, an endemic area of Triatoma braziliensis, in the farm of Boa Vista. Bug capture in the area revealed that of 171 T. braziliensis $48.8 \%$ were infected with Trypanosoma cruzi. Only five Triatoma pseudomaculata were caught. Of the 250 inhabitants of this farm $7.7 \%$ had positive serology for $T$. cruzi infection (indirect immunofluorescent test). $9.1 \%$ of dogs had positive xenodiagnoses.

In the house to be demolished one inhabitant had positive serology but $26 T$. braziliensis captured before dmolition were all uninfected. During demolition an rat infected with $T$. cruzi was found and of 104 triatomine bugs $89.4 \%$ were T. pseudomaculata and $10.6 \% T$. braziliensis. Infected Rhodnius nasutus was found in the chicken house.

Examples of $T$. pseudomaculata were found in the roof $(0.6$ per swquare metre)and in the external an internal walls of the living room 10.8 per square metre). Precipitin tests of blood meals revealed marked anthrophilia.

We believe this house to have been initially colonised by $T$. braziliensis. After sprying this species was substituted by $T$. pseudomaculata a species feeding on man with a infection rate sufficient to effect transmission.
AGRADECIMENTOS - Agradecemos a todos aqueles que direta ou indiretamente colaboraram para tornar possível a realização deste trabalho. São muitas as pessoas e a referência a alguns poderia levar-nos a uma omissão injusta de nome.

\section{REFERẼNCIA BIBLIOGRÁFICAS}

1. Alencar, J.E. - Estudos sobre a epidemiologia da doença de Chagas no Ceará. III Região do Baixo Jaguaribe. Rev. Bras. Malariol. D. Trop., $17(2,3)$ : $149-156,1965$.

2. Alencar, J.E. - Almeida, Y.M., Santos, A.R. \& Freitas, L.M. - Epidemiology of Chagas disease in the State of Ceará, Brasil - Rev. Bras. Malariol. D. Trop. 26, $27: 5-26,1975$.

3. Alencar, J.E., Santos, A.R., Bezerra, O.F. \& Saraiva, T.M. - Distribuição geográfica dos principais vetores de endemias no Estado do Ceará. 1 - Triatomíneos. Rev. Soc. Bras. Med. Trop., 10 (5): $261-284,1976$

4. Alencar, J.E., Almeida, Y.M., Freitas, L. M. \& Santos, A.R. - Estudos sobre a epidemiologia da doenças de Chagas no Estado do Ceará - Brasil - VI Estudos em uma micro-área de Russas. Em Publicação na Rev. Soc. Bras. Med. Trop., 1976. 